\title{
European Union Drug Regulating Authorities Clinical Trials Database
}

National Cancer Institute

\section{Source}

National Cancer Institute. European Union Drug Regulating Authorities Clinical Trials

Database. NCI Thesaurus. Code C132782.

The European Union's electronic database of clinical trials. It contains information submitted by sponsors and informs users about ongoing clinical trials in EU Member States and European Economic Area countries. 